\title{
Avaliação das pacientes com câncer de mama submetidas a cirurgia oncológica na Santa Casa de Franca no período de janeiro/2015 a fevereiro/2018
}

Evaluate breast cancer patients' profiles who were undergone oncologic surgery at Santa Casa de Franca hospital complex from January/2015 to February/2018

Evaluación de las pacientes con cáncer de mama sujeta a cirugía oncológica en la Santa Casa de Franca en el período de Enero/2015 a Febrero/2018

Clarissa Barbarotto ${ }^{1 *}$, Isabella Fioretti Achitti ${ }^{1}$, Priscilla Lima Martins ${ }^{1}$, Karine Angélica Cintra ${ }^{1,2}$.

\section{RESUMO}

Objetivo: Avaliação do perfil de pacientes com câncer de mama submetidas a cirurgia oncológica no complexo hospitalar da Santa Casa de Franca no período de janeiro/2015 a fevereiro/2018. Resultados: 170 mulheres com câncer de mama foram submetidas à cirurgia oncológica no período estudado. A idade média foi de 59,9 anos; sendo que o maior número de pacientes foi submetida a cirurgia entre 61 e 70 anos (36,1\%). Em relação a paridade e raça, $53,3 \%$ multigestas e $78,1 \%$ brancas. A mastectomia radical modificada foi realizada em $55,6 \%$ dos casos e a cirurgia conservadora em $44,4 \%$. O tipo histológico predominante foi o ductal ( $90 \%$ dos casos). Ao compararmos o estadiamento clínico das pacientes com avaliação patológica do tamanho do tumor $(\mathrm{T})$ e extensão linfonodal $(\mathrm{N})$, a média da diferença mostrou uma subestima do exame físico com exame histológico. Conclusão: A análise realizada no presente estudo demonstrou semelhança com os dados descritos na literatura em respeito às características populacionais de pacientes com câncer de mama, exceto, a discordância em relação à multiparidade, considerada como fator protetor. Além disso, observou-se maior realização de mastectomias em pacientes abaixo dos 50 anos.

Palavras-chaves: Câncer de mama, Cirurgia conservadora da mama, Mastectomia.

\begin{abstract}
Objective: Evaluate breast cancer patients' profiles who were undergone oncologic surgery at Santa Casa de Franca hospital complex from January/2015 to February/2018. Results: 170 women with breast cancer underwent oncologic surgery within the period studied. The average age was 59,9 years; and most of patients underwent surgery between 61 and 70 years (36,1\%). Regarding to parity and race, 53,3\% multigested and $78,1 \%$ white. The modified radical mastectomy was performed in $55,6 \%$ of the cases and conservative surgery in $44,4 \%$. The predominant histological type was the ductal ( $90 \%$ of the cases). When comparing the clinical staging of the patients to pathological evaluation of tumor size $(\mathrm{T})$ and lymph node extension $(\mathrm{N})$, the average difference showed an underestimation of the physical examination with the histological one. Conclusion: The analysis performed in this study proved similarity with the data described in the literature in relation to the population characteristics among breast cancer patients, except for the disagreement concerning multiparity, regarded as a protective factor. In addition, greater mastectomies were observed in patients under 50 years of age.
\end{abstract}

Key words: Breast cancer, Traditional breast surgery, Mastectomy.

\footnotetext{
${ }^{1}$ Universidade de Franca (UNIFRAN), Franca-SP. *E-mail: clerbarbarotto@hotmail.com

${ }^{2}$ Santa Casa de Franca, Franca-SP.
}

SUBMETIDO EM: 5/2019 


\section{RESUMEN}

Meta: Evaluación del perfil de pacientes con cáncer de mama sujeta a cirugía oncológica en el complejo hospitalario de la Santa Casa de Franca en el período de enero / 2015 a febrero / 2018. Resultados: 170 mujeres con cáncer de mama fueron atendidas en el período estudiado. La edad media fue de 59,9 años; y el $36,1 \%$ de las pacientes fueron sometidas a la cirugía oncológica las cuales presentaban una edad de 61 a 70 años. En relación a paridad y raza, $53,3 \%$ eran mujeres con más de un parto y $78,1 \%$ blancas. La mastectomía radical modificada fue realizada en $55,6 \%$ de los casos y la cirugía conservadora en $44,4 \%$. El tipo histológico imperante fue el ductal ( $90 \%$ de los casos). Al comparar la estadística clínica de las pacientes con la evaluación patológica del tamaño del tumor $(\mathrm{T})$ y extensión del ganglio linfático $(\mathrm{N})$, la media de la diferencia mostró una subestimación del examen físico con el examen histológico. Conclusión: El análisis realizado en el presente estudio demostró semejanza con los datos descritos en la literatura en relación a las características poblacionales de pacientes con cáncer de mama, excepto, la discordancia en relación a la multi-paridad, considerada como factor protector. Además, se observó mayor realización de mastectomías en pacientes menores de los 50 años.

Palabras-clave: Cáncer de mama, Cirugía de la mama; Mastectomía.

\section{INTRODUÇÃO}

O câncer de mama é o segundo tipo mais frequente de neoplasia que acomete mulheres, ficando atrás apenas do câncer de pele não melanoma, tanto em países desenvolvidos quanto em países em desenvolvimento (BREGAGENOL RK e DIAS AS, 2010; FRAZÃO A e SKABA MMFV, 2013).

Há grande preocupação com este tipo de neoplasia, já que o número de casos vem aumentando a cada ano, e corresponde a $25 \%$ de todas as neoplasias (1,67 milhão casos novo/ano) no mundo e $22 \%$ dos casos novos no Brasil, sendo, na população feminina, a principal causa de morte por neoplasia maligna (BREGAGENOL RK e DIAS AS, 2010; SALIMENA AMO, et al., 2012).

Ele acomete principalmente mulheres na perimenopausa, por estar intimamente relacionado ao avanço da idade e às alterações hormonais, sendo mais raro em jovens (PINHEIRO AB, et al., 2013).

Em relação aos países desenvolvidos, segundo a American Cancer Society (2016 e 2017) estimou em 2018 nos Estados Unidos 226 mil novos casos de cânceres invasivos em mulheres, 63 mil novos casos de carcinoma in situ e mortalidade de 40 mil mulheres. Nos países europeus as estimativas se equivalem, de acordo com a Globocan (2012). Umas das particularidades dos países em desenvolvimento, incluindo o Brasil, é o aumento proporcionalmente maior na incidência de mulheres entre 40 e 50 anos comparado ao reportado em países desenvolvidos (URBAN LABD, et al., 2017).

Os estudos de Frazão A e Skaba MMFV (2013), o Registro de Câncer de Base Populacional (RCBP) apontou que, no Brasil, entre os anos de 2000 e 2008, 46,2\% dos cânceres de mama estavam em estadiamento avançado (estádios III e IV). Este fato pode ser atribuído à própria história natural da doença ou pela falha no diagnóstico precoce do Sistema Único de Saúde (SUS).

São utilizadas duas ferramentas para a detecção precoce do Câncer de mama: o diagnóstico precoce e o rastreamento. O primeiro trata-se da abordagem dos sinais e sintomas clínicos das pacientes; já no segundo é realizado o exame de mamografia em pacientes assintomáticas. No Brasil, segundo o site do Instituto Nacional de Câncer (INCA) (2018), o Ministério da Saúde recomenda que esse rastreio seja realizado entre 50 e 74 anos e a Federação Brasileira das Associações de Ginecologia e Obstetrícia (FEBRASGO) dos 40 aos 74 anos.

A fisiopatologia da doença é multifatorial. Engloba fatores de risco relacionados com idade, características genéticas (mutações nos genes BRCA1\BRCA2, relacionados a supressão de tumores através de atuações nos processos celulares, como controle do ciclo celular), hábitos de vida (alimentação, tabagismo, etilismo), meio ambiente, hábitos reprodutíveis, duração da atividade ovariana e uso de medicamentos (em especial 
terapia hormonal após a menopausa por longo período) (BREGAGENOL RK e DIAS AS, 2010; PINHEIRO $A B$, et al., 2013). Para o diagnóstico da doença, além do exame físico e da mamografia, pode-se também utilizar a ultrassonografia para complementar a investigação quando necessário, e no auxílio para a realização de biópsia, estadiamento clínico e definição da terapêutica (SOUZA NHA, et al., 2017).

Os métodos mais utilizados para o diagnóstico histológico são a biópsia por agulha grossa ou core biopsy e a biópsia cirúrgica. A core biopsy pode ser realizada em lesões palpáveis e não palpáveis, podendo ser guiadas por ultrassonografia ou mamografia. Sua vantagem é obter material histológico adequado para o diagnóstico do câncer e permitir o planejamento cirúrgico posterior. Já a biópsia cirúrgica é utilizada quando há divergências entre os achados clínicos e a biópsia por agulha grossa ou quando o resultado é questionável (BEREK JS, 2014).

Dentre os tipos histológicos do câncer de mama destacam-se o ductal (aproximadamente 70\% dos casos, com diversos subtipos) e os lobulares (30\%), podendo ser invasivos ou não invasivos (também denominados carcinomas in situ, que não ultrapassam a membrana basal) (BEREK JS, 2014).

O tratamento cirúrgico do tumor pode ser realizado de forma radical ou conservadora. Na primeira opção, realiza-se a mastectomia e, na segunda, a quadrantectomia com margens de segurança oncológica. Ambos podem ou não estar associados ao esvaziamento axilar ou pesquisa do gânglio sentinela. De acordo com a Portaria oㅡ 1008 do Ministério da Saúde (2015), as indicações dependem do estadiamento tumoral, volume mamário e características de cada paciente, como idade, presença de comorbidades e preferência da mulher.

O estadiamento, foi criado pela União Internacional Contra o Câncer (UICC), em que se utiliza como parâmetros para classificação do tumor: dimensão do tumor $(T)$, extensão aos linfonodos $(N)$ e presença ou não de metástases (M) (FRAZÃO A e SKABA MMFV, 2013).

Após o tratamento cirúrgico preconiza-se alcançar um controle local e sistêmico da doença com o tratamento adjuvante, com o objetivo de diminuir o risco de recorrência da doença e prolongar a sobrevida das pacientes. Dentre as modalidades estão: a quimioterapia, hormonioterapia e radioterapia. A decisão quanto a esse tratamento deve ser baseada considerando-se o estadiamento e os riscos existentes.

Dentre os efeitos colaterais descritos destacam-se os déficits neurocognitivos crônicos após quimioterapia, ressecamento vaginal e fogachos no tratamento sistêmico com tamoxifeno e artralgias relacionadas aos inibidores de aromatase. Quanto à radioterapia, deve ser realizada após todas as cirurgias conservadoras e, segundo as diretrizes da American Society of Clinical Oncology, após a mastectomia em mulheres com tumor primário $>5 \mathrm{~cm}$ e/ou com quatro ou mais linfonodos axilares positivos; invasão vascular ou de pele (BEREK JS, 2014).

O presente estudo tem como objetivo avaliar o perfil das pacientes com câncer de mama submetidas a cirurgia oncológica no Complexo Santa Casa de Franca, observando dados demográficos (idade, procedência e estado civil), características reprodutivas, estadiamento clínico do tumor e estadiamento patológico.

\section{MATERIAIS E MÉTODOS}

Foram revisados os prontuários das pacientes com diagnóstico de Câncer de mama que foram submetidas à cirurgia oncológica no complexo hospitalar da Santa Casa de Franca - SP, no período de janeiro de 2015 a fevereiro de 2018. Foram incluídas todas as pacientes com câncer de mama submetidas a cirurgia oncológica no período descrito.

As variáveis analisadas foram: idade, raça, paridade, estadiamento clínico e patológico do tumor [segundo o Sistema TNM, o qual avalia tumor primário $(T)$, linfonodos $(N)$ e metástase $(M)$ ], tipo histológico e o tipo de cirurgia realizada.

As variáveis numéricas foram descritas pelos parâmetros de média aritmética e desvio padrão e a condução dos testes estatísticos foi na perspectiva não paramétrica. O nível de significância pré-fixado foi de 5,0\% $(p<0,05)$ e os testes estatísticos foram realizados no software GraphPad Prism 5.0. 
Os gráficos descritivos foram confeccionados no software Excel / Office 2010. O estudo foi aprovado pelo Comitê de Ética e Pesquisa da Santa Casa de Misericórdia de Franca - SP sob o número de registro CAAE $n^{\circ} 01445918.2 .0000 .5438$.

\section{RESULTADOS}

Foram avaliadas 170 mulheres com diagnóstico de câncer de mama que foram submetidas à cirurgia oncológica no complexo Santa Casa de Franca, no período de janeiro/2015 a fevereiro/2018. A idade média das mulheres no estudo foi de 59,9 anos; sendo o maior número de cirurgias realizado em pacientes na faixa etária entre 61 a 70 anos (36,1\%). Nas demais idades, a percentagem de cirurgias foram, respectivamente, $4,1 \%, 14,8 \% 30,8 \%$ e 14,2\% nas idades $\leq 40$ anos, entre 41 e 50 anos, 51 e 60 anos e $>70$ anos (Gráfico 1).

Gráfico 1- Faixa etária das pacientes com Câncer de Mama submetidas a cirurgia oncológica, n=170. Franca-SP, 2019.

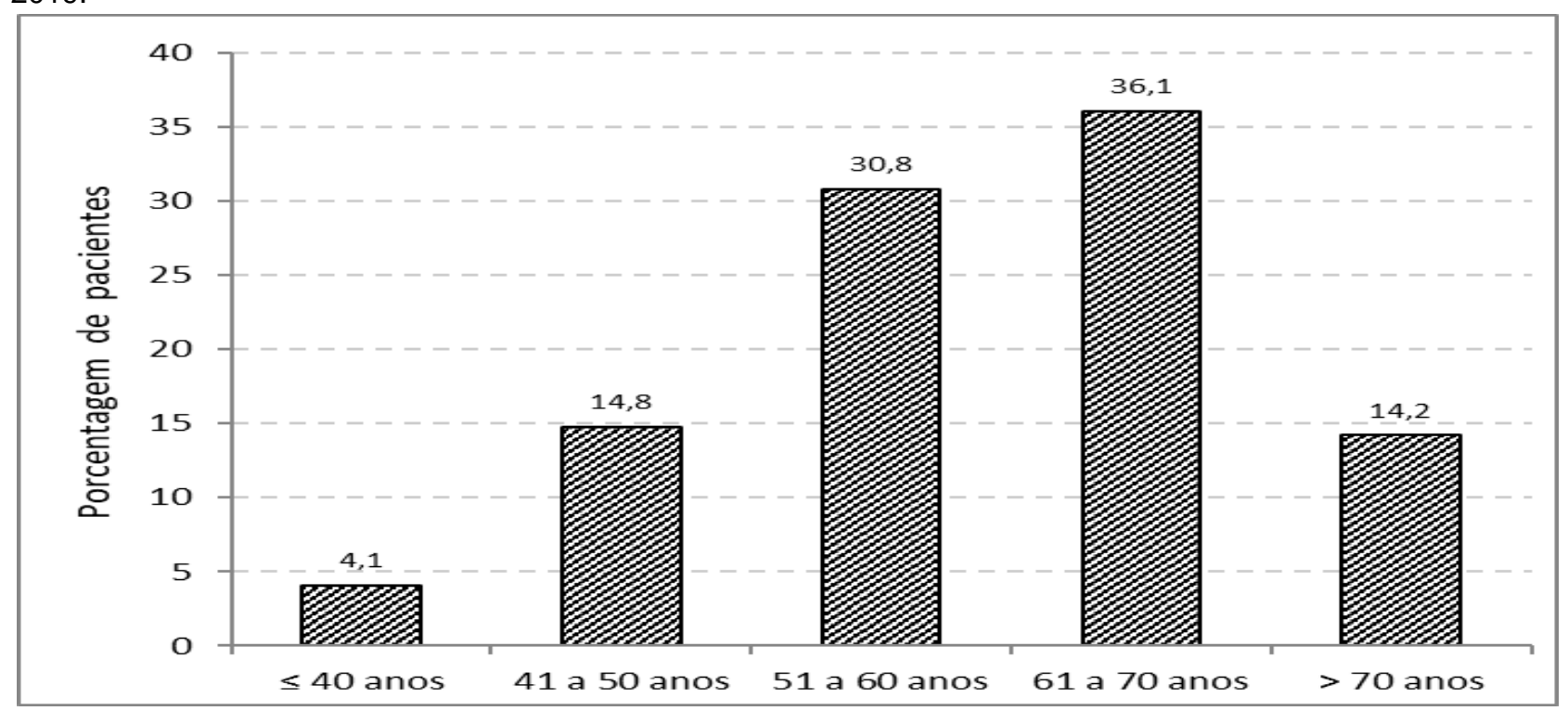

Fonte: Dados da pesquisa, 2019.

Gráfico 2 - Paridade das pacientes com Câncer de Mama submetidas a cirurgia oncológica, n=170. Franca-SP, 2019.

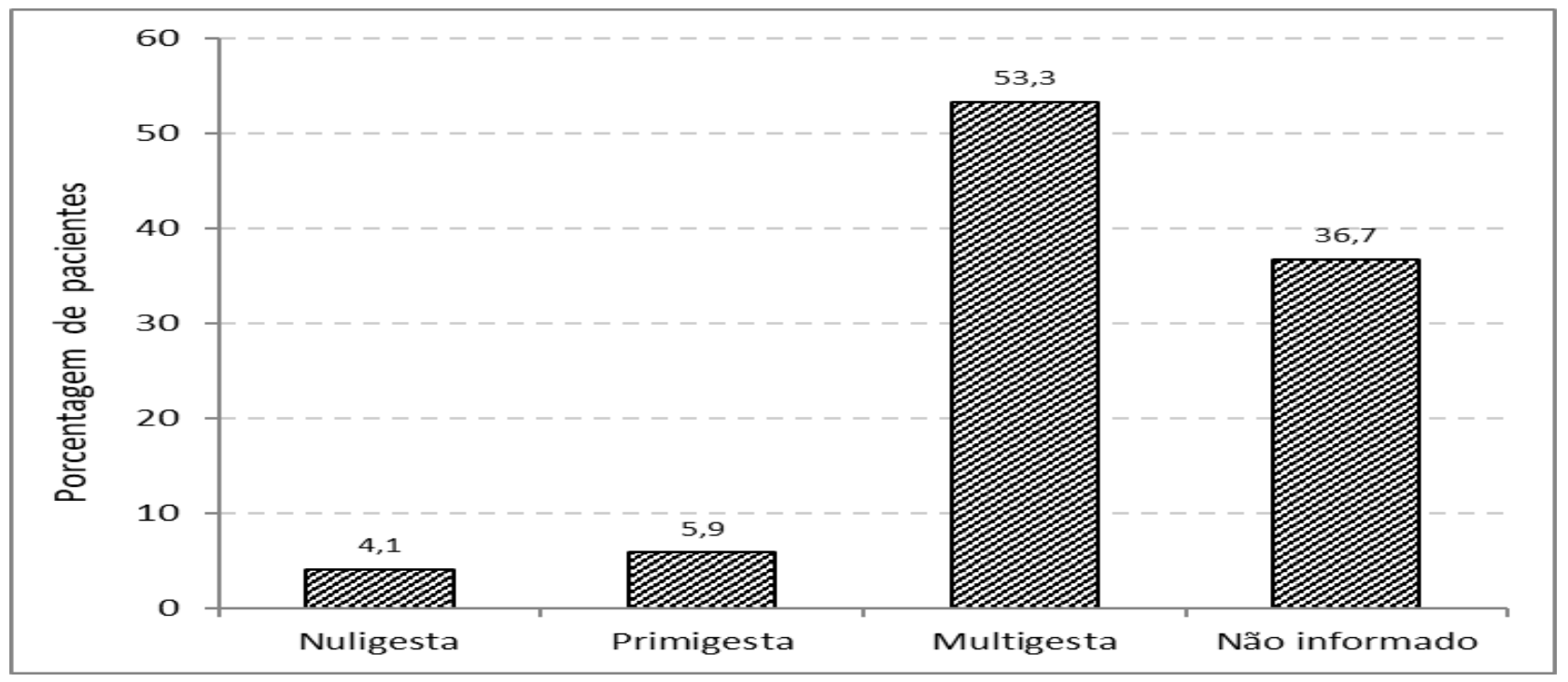

Fonte: Dados da pesquisa, 2019. 
A análise da paridade demonstrou maior prevalência da doença em multigestas (53,3\% das pacientes), seguidas de paridade não informada em prontuário (36,7\%) primigesta (10\%) e nuligestas $(4,1 \%)$. O número médio de filhos das mulheres estudadas foi de 3,3 \pm 2,6 (DP). (Gráfico 2).

Ao observarmos a raça, observou-se que $78,1 \%$ das mulheres diagnosticadas consideravam-se da cor branca, $13,6 \%$ negras e $8,3 \%$ pardas (Gráfico 3).

Gráfico 3 - Raça das pacientes com Câncer de Mama submetidas a cirurgia oncológica, $n=170$. Franca-SP, 2019.

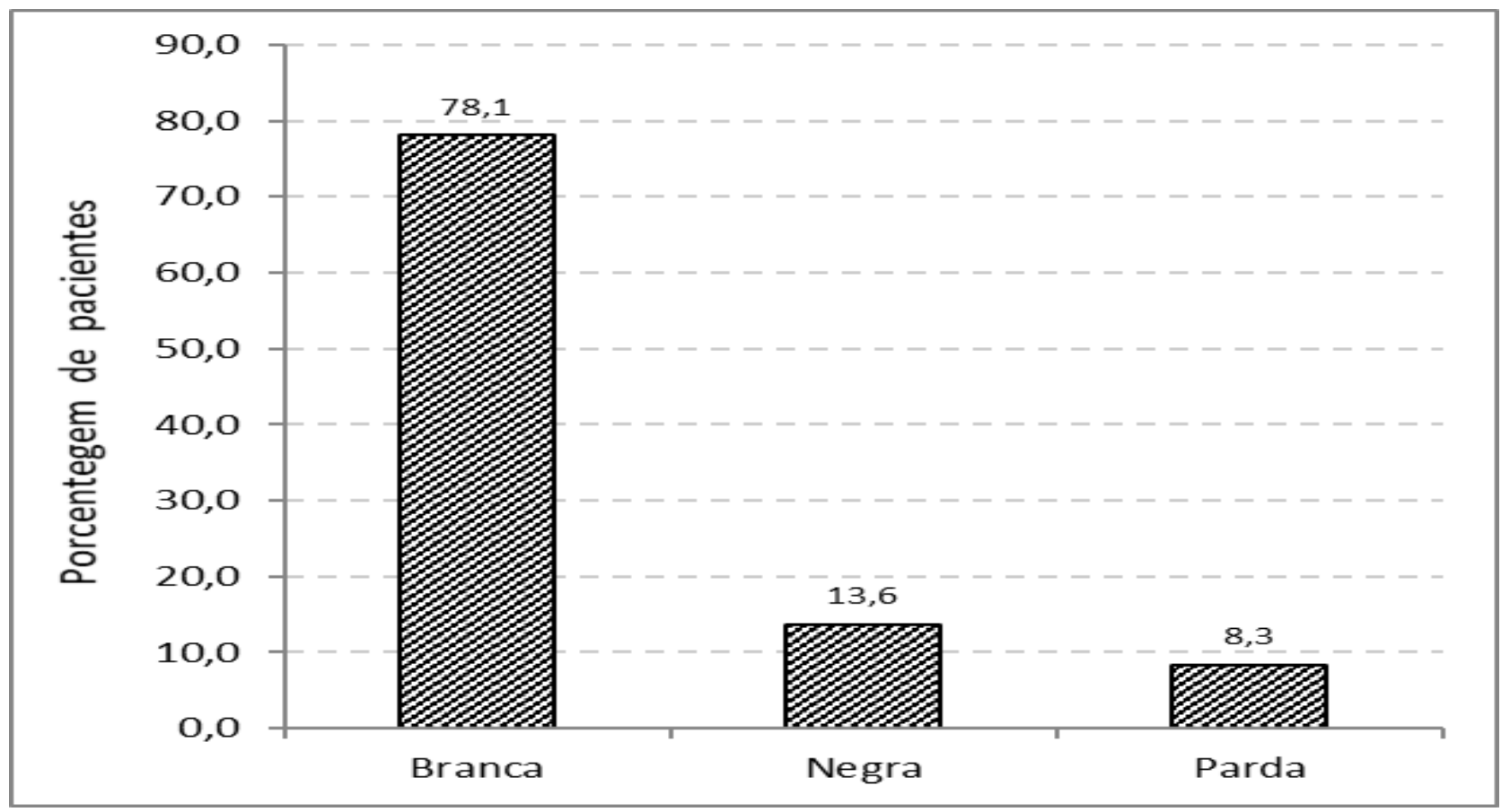

Fonte: Dados da pesquisa, 2019.

A mastectomia radical foi realizada em $55,9 \%$ dos casos e a cirurgia conservadora em $44,1 \%$ (Gráfico 4 ).

Gráfico 4 - Tipo de cirurgia das pacientes com Câncer de Mama submetidas a cirurgia oncológica, $n=170$. Franca-SP, 2019.

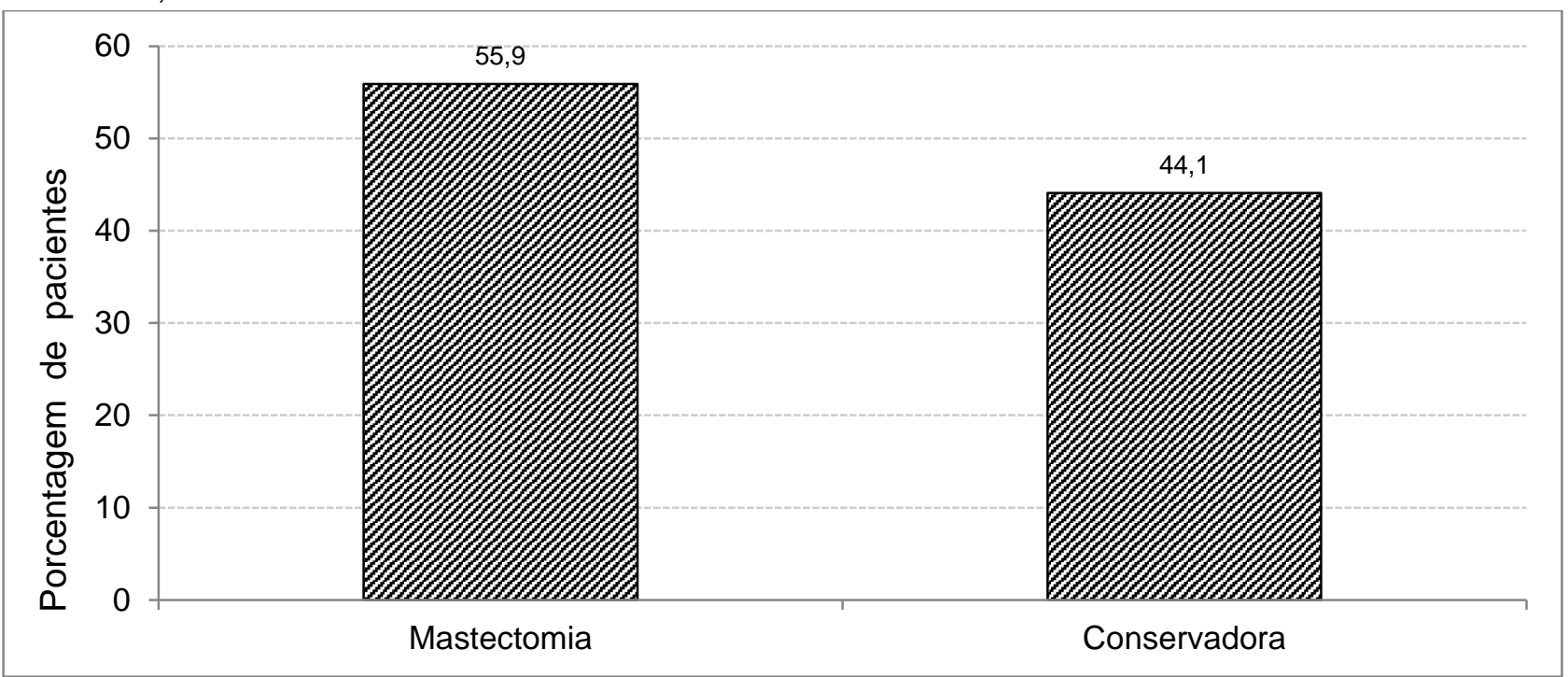

Fonte: Dados da pesquisa, 2019. 
Referente ao estudo histopatológico dos tumores, houve predominância do tipo ductal, que representou $90,5 \%$ do total, seguido do tipo lobular (7,1\%) e outros tipos em 2,4\% (Gráfico 5).

Gráfico 5 - Subtipos histopatológicos das pacientes com Câncer de Mama submetidas a cirurgia oncológica, n=170. Franca-SP, 2019.

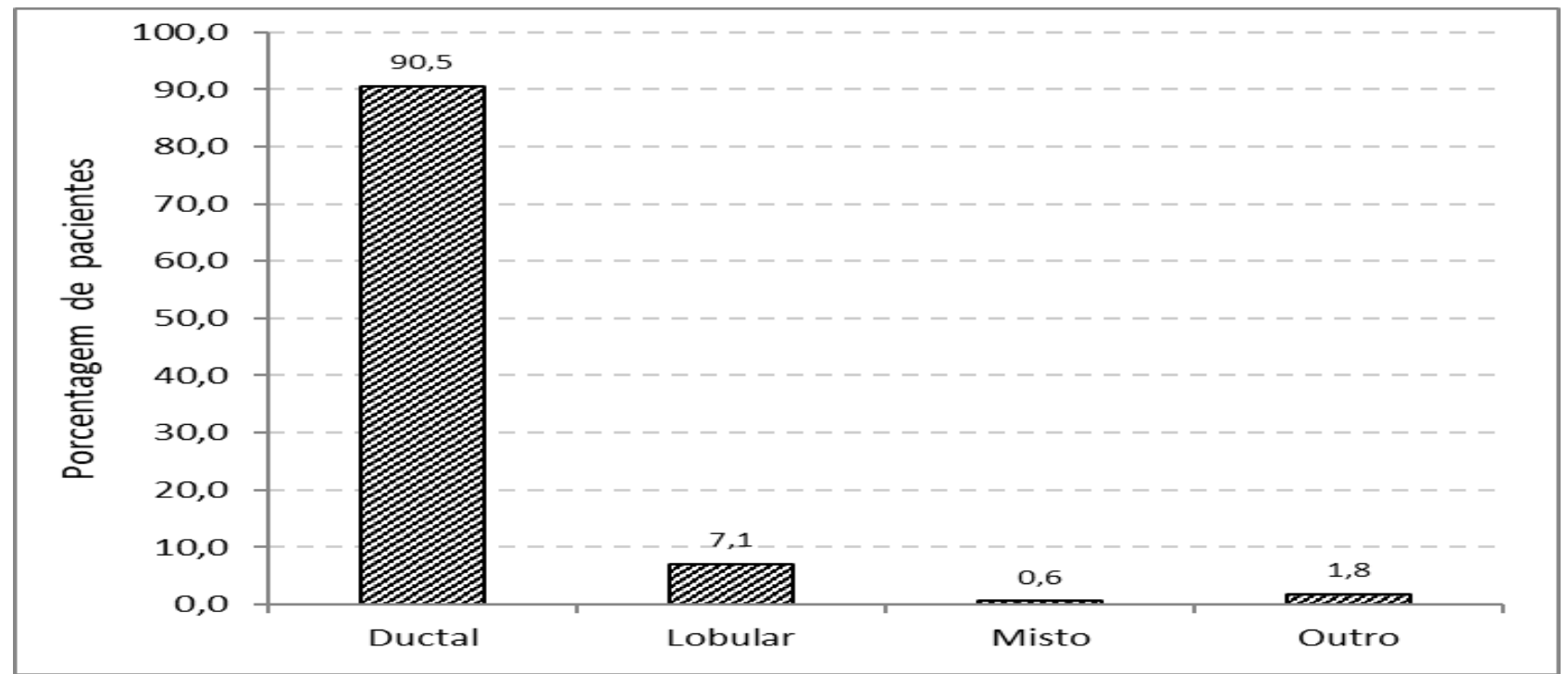

Fonte: Dados da pesquisa, 2019.

As pacientes na faixa etária entre 51 e 70 anos apresentaram alta prevalência de tumores classificados como T2, com $32,7 \%$ dos casos ( $p<0,0001$ ), quando comparadas às outras faixas etárias e as mulheres entre 61 e 70 anos, apresentaram o maior número de tumores classificados como T4, representando $9,6 \%$ do total $(\mathrm{p}<0,0001$ (Gráfico 6).

Gráfico 6 - Estadiamento do tumor em relação com a idade em pacientes submetidas a mastectomia.

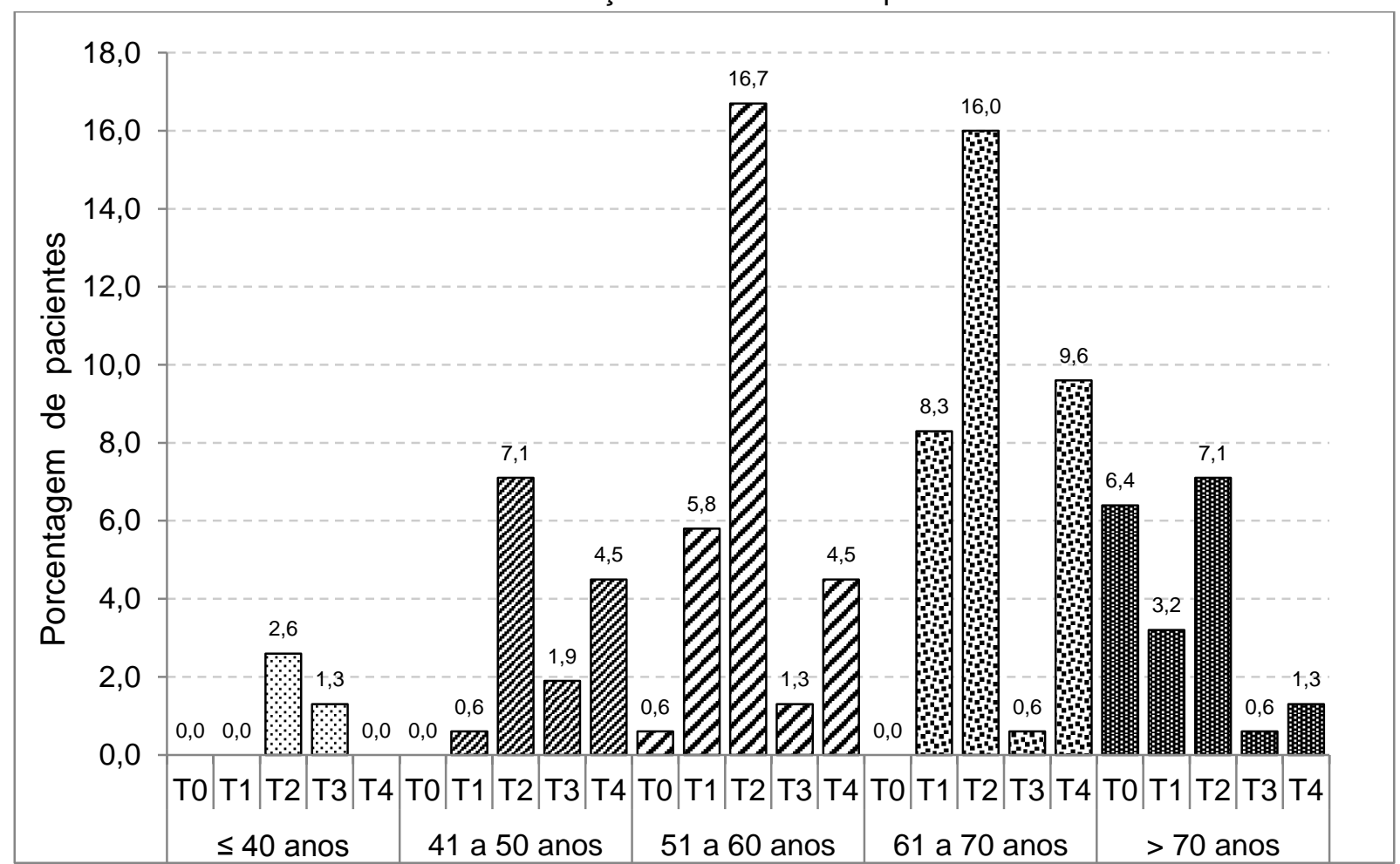

Fonte: Dados da pesquisa, 2019. 
Gráfico 7 - Extensão linfonodal em relação a idade em pacientes submetidas a mastectomia.

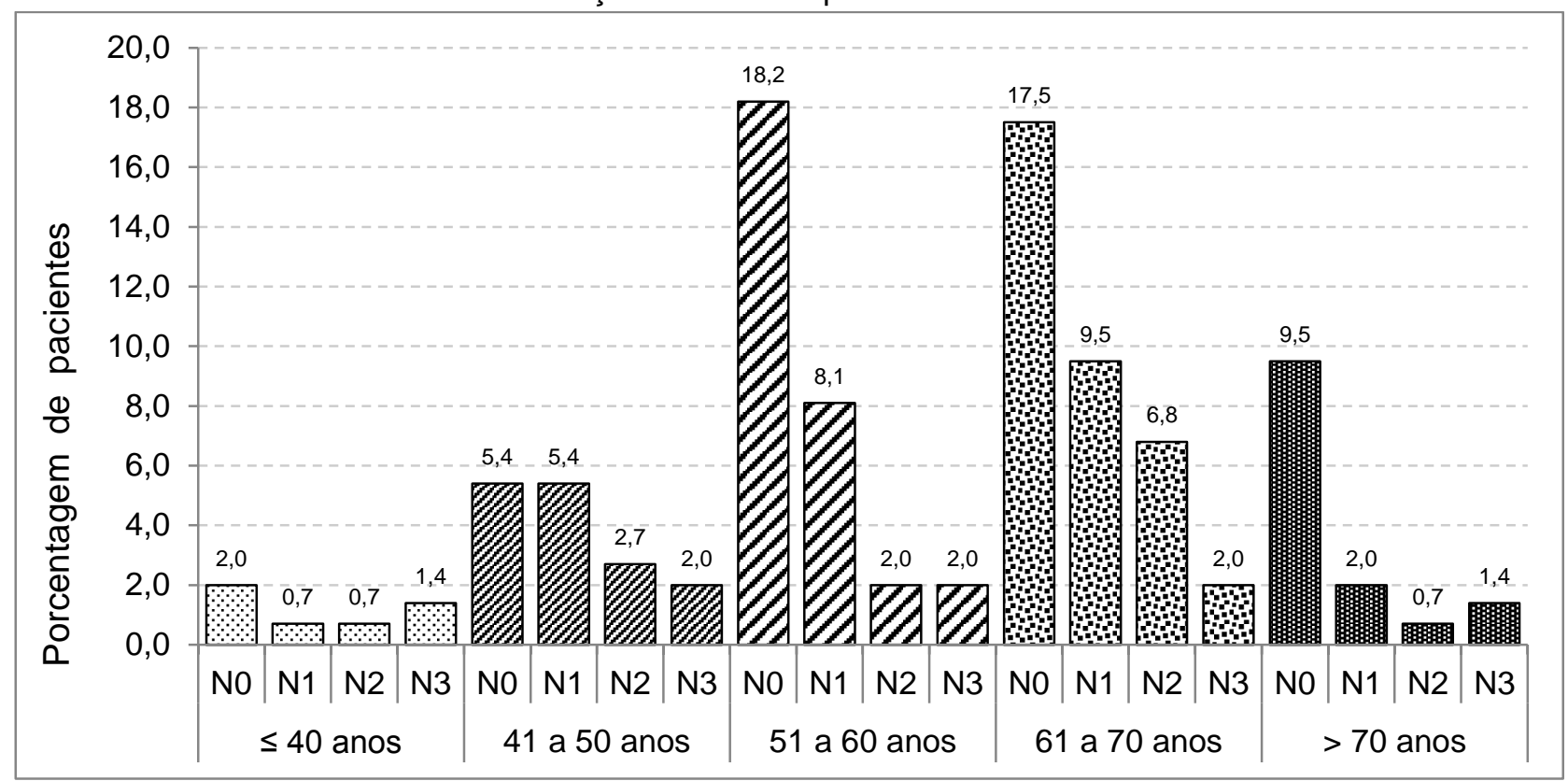

Fonte: Dados da pesquisa, 2019.

Gráfico 8 - Presença de metástase em relação a idade em pacientes submetidas a mastectomia.

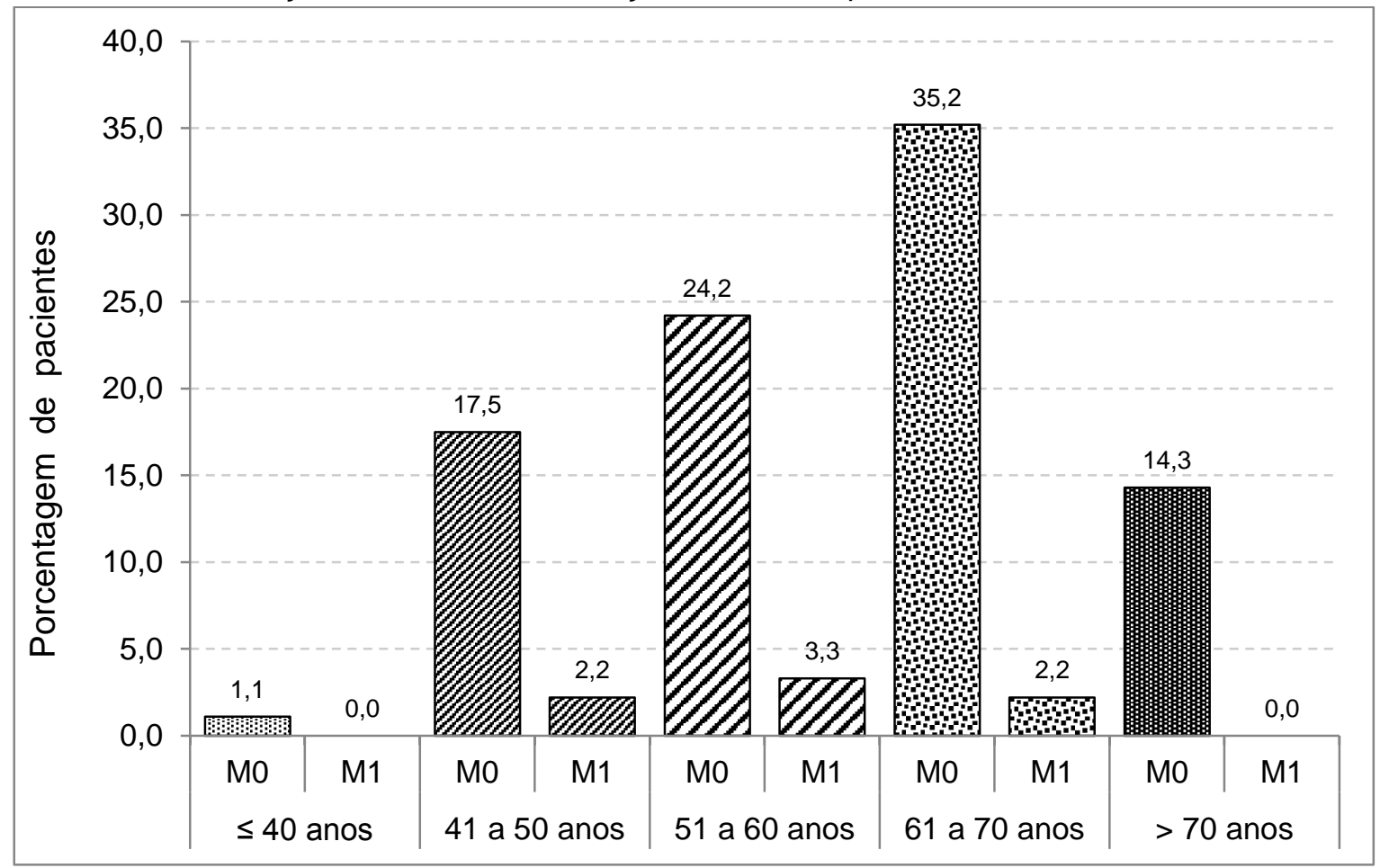

Fonte: Dados da pesquisa, 2019.

A comparação entre o estadiamento clínico e o estadiamento patológico, feito através do Teste de Kappa, das pacientes demonstrou uma subestimação da extensão da doença clínica, tanto em relação ao tamanho do tumor $(T)(p=0,0053)$, como da extensão para linfonodos $(N)(p<0,0001)$.

Também foi feita a associação entre o procedimento cirúrgico e as idades das pacientes, em que foi evidenciado uma prevalência significativa de mastectomias nas pacientes com faixas etárias de até 50 anos ( $p$ $=0,0047$ ). Nas demais faixas etárias não houve diferença estatisticamente significativa (Gráfico 9). 
Gráfico 9 - Relação do tipo cirúrgico e idade das pacientes com Câncer de Mama submetidas a cirurgia oncológica, $\mathrm{n}=170$. Franca-SP, 2019.

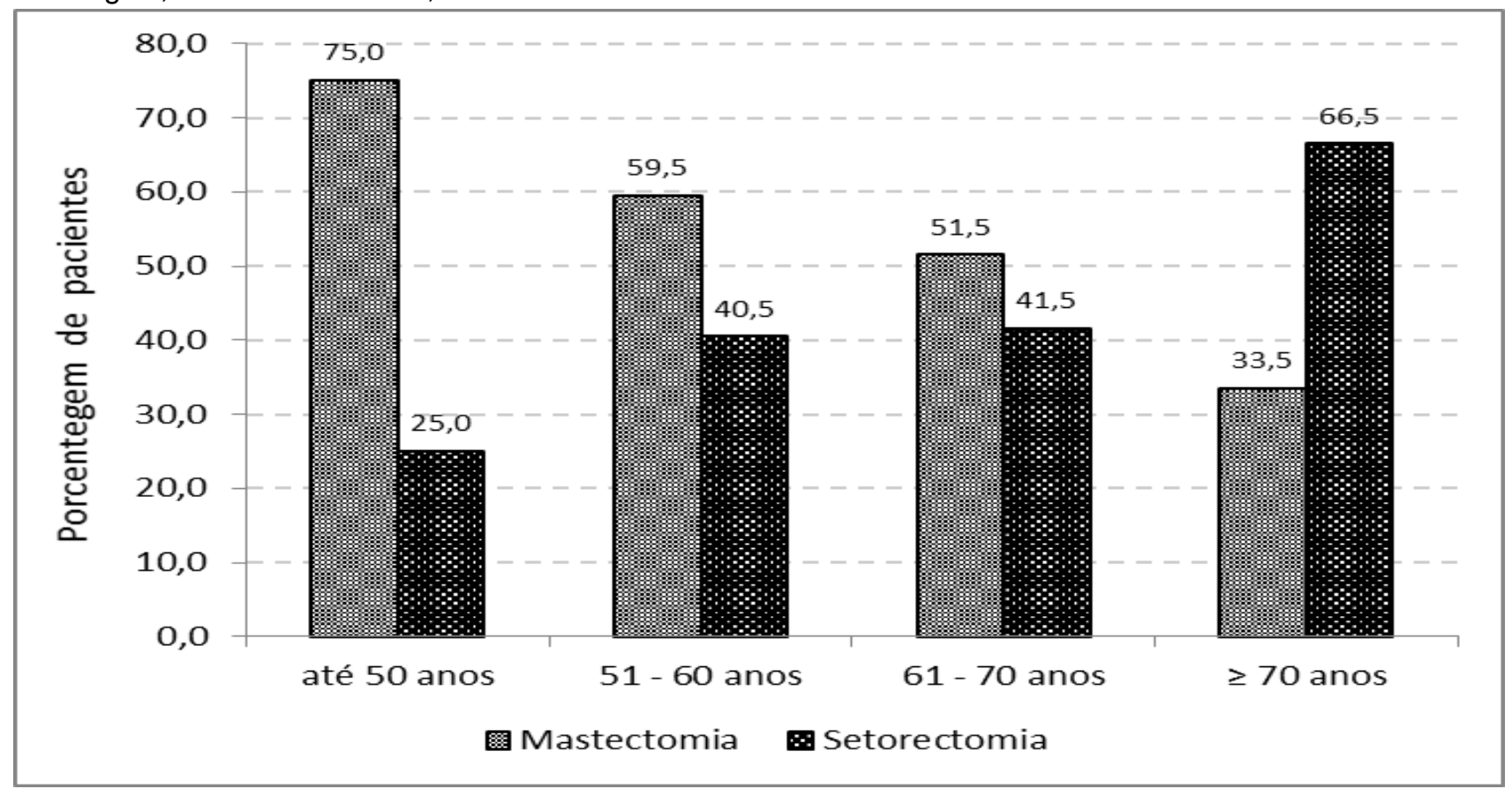

Fonte: Dados da pesquisa, 2019.

Observou-se, ainda, uma redução significativa de mastectomias $(p=0,0397)$ e um aumento expressivo das setorectomias $(p=0,041)$ na medida em que a idade das pacientes aumentou (Gráfico 10). As demais variáveis analisadas não apresentaram significância estatística.

Gráfico 10- Relação entre tipo cirúrgico e idade de pacientes com Câncer de mama e submetidas a mastectomia.

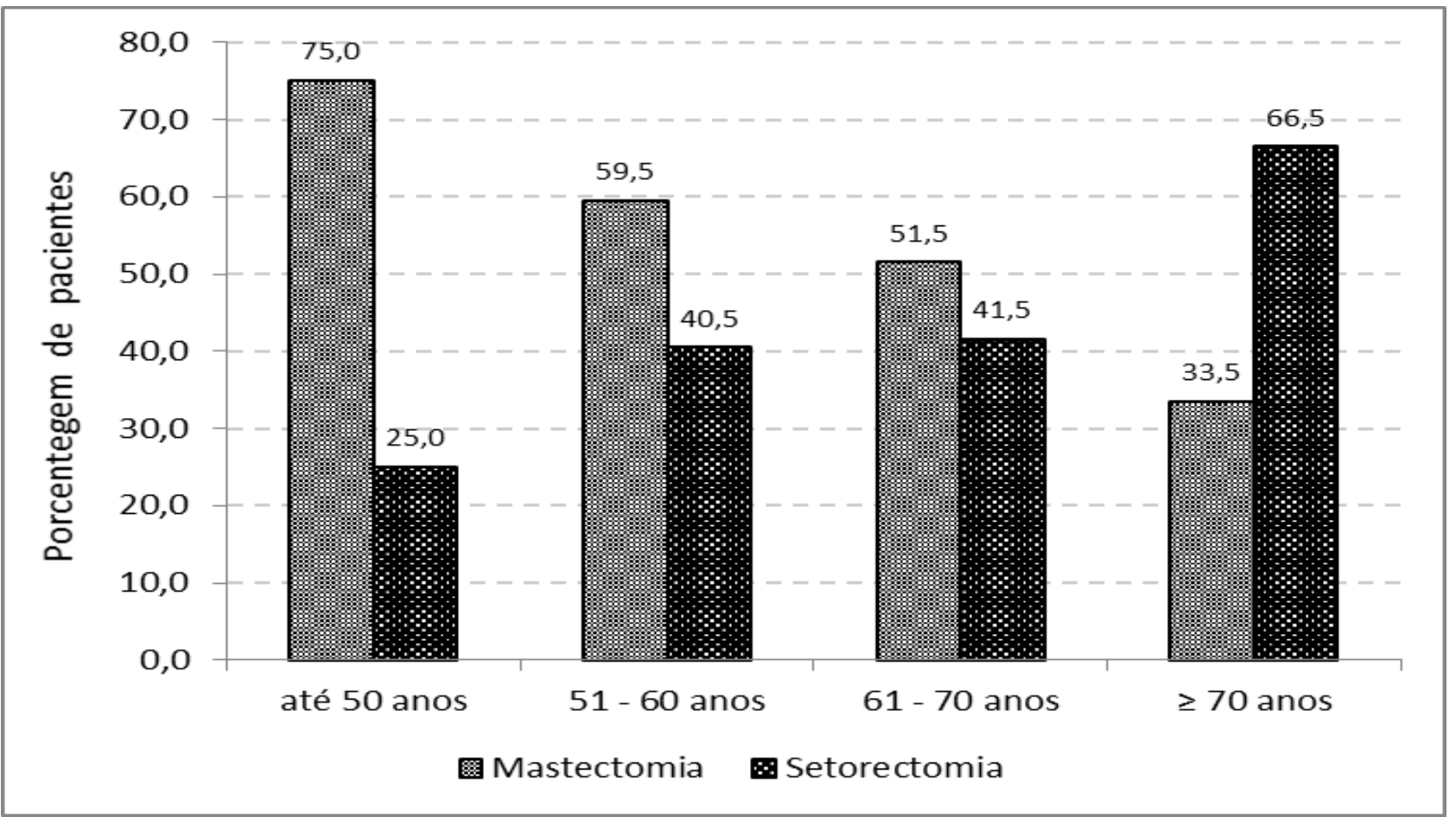

Fonte: Dados da pesquisa, 2019. 


\section{DISCUSSÃO}

Os dados do estudo foram compatíveis com os dados da literatura, já que demonstrou que a idade é um fator de risco para a doença. As características da amostra estudada apresentaram idade média da doença um pouco superior às relatadas em estudos semelhantes na literatura. A maioria das mulheres submetidas a cirurgia oncológica, neste estudo, estavam na faixa etária entre 61 e 70 anos, com idade média de 59,9 anos (Gráfico 1).

Em estudo de Azevedo BA, et al. (2017), realizado no estado de Goiás, essa idade média foi de 56 anos, em análise no período dos anos de 2003 a 2013. Além disso, outros estudos realizados no sul e sudeste do Brasil por Munhoz CAS (2009) e Tessaro S, et al. (2001), observaram idade média de 53 a 53,7 anos, respectivamente.

Ao analisar o estadiamento, nos países desenvolvidos o diagnóstico do câncer ocorre em estadios clínicos mais precoces (T1 e T2), sem extensão linfonodal ou metastática, correspondendo a 80\% dos casos de neoplasia mamária. Como exemplo, na Itália, apenas $10 \%$ dos tumores recebem o diagnóstico em estádios T3 ou T4 (PAULINELLI RR, et al., 2003).

No Brasil, um estudo de Thuler LCS e Mendonça GA (2005), publicado na Revista Brasileira de Ginecologia que analisou as mulheres com tumor de mama atendidas no SUS, observou a maior parte dos casos no estadio II $(42,8 \%)$ e III $(32,6 \%)$. Outro trabalho, realizado no nordeste brasileiro, encontrou $34,5 \%$ das mulheres no estadio II (8 -18\%).

Dados coletados do estado de São Paulo em 2006, do registro hospitalar de câncer da região, expõem o maior percentual de diagnóstico no estadio II, com $40 \%$ dos casos. O A.C. CAMARGO CANCER CENTER (2019) em dados do seu site, também demonstrou alto percentual de diagnósticos estágio II (23,53\%).

No presente estudo, a maior parte dos diagnósticos dos tumores eram de tamanho entre 2 e $5 \mathrm{~cm}$ (T2) no estadiamento, em mulheres de 51-70 anos ( $p<0,0001)$ (Gráfico 6), o que é compatível com a literatura brasileira, demonstrando uma identificação precoce das pacientes atendidas no SUS, com melhores possibilidades de tratamento.

Uma análise estatisticamente significativa realizada neste estudo, pelo Teste de Kappa, demonstrou que o estadiamento clínico do exame físico é subestimado em relação a avaliação patológica, no que diz respeito ao tamanho $(T)(p=0,0053)$ e a extensão linfonodal $(N)(p<0,0001)$ do tumor.

Tal fato pode ter ocorrido devido a demora na abordagem cirúrgica das pacientes diagnosticadas. Como salientado acima, a identificação das pacientes com tumores de mama está ocorrendo de forma cada vez mais precoce, ao contrário da cirurgia que demora a acontecer, ocorrendo o aumento do tamanho do tumor no momento da exérese em relação ao primeiro exame físico.

Já é conhecido mundialmente que um dos fatores de risco associados ao câncer de mama é a nuliparidade e, segundo Freitas F, et al., (2011), isso se deve à ação prolongada do hormônio estrogênio. Contudo, na análise presente, esse dado foi controverso, já que apresentou a maioria das mulheres com mais de uma gestação (53,3\%) (Gráfico 2). Deve-se salientar que houve um grande número de falha de registo no prontuário médico $(36,7 \%)$, o que pode ter influenciado essa análise.

Estudo de Paiva CE, et al. (2002), publicado na Revista Brasileira de Cancerologia estimou uma diminuição de $10 \%$ no risco de desenvolvimento de câncer de mama para cada gestação completa adicional. A discussão da nuliparidade como fator de risco evidencia que o atraso na primeira gestação auxilia o processo de maturação das células da mama, tornando-as potencialmente mais protegidas em relação à ação de substâncias cancerígenas (PINHARD CR e MERCÊS NNA, 2009).

Ao analisarmos a raça, observou-se que $78,1 \%$ das mulheres diagnosticadas consideravam-se da cor branca, $13,6 \%$ negras e 8,3\% pardas. Esse dado está em concordância com a literatura (Gráfico 3). Em estudo feito em Pernambuco, $60 \%$ das mulheres se autorreferiram como de cor branca (PAIVA CJK e CESSE EAP, 2015). 
Assim como cor branca teve o maior percentual de mortalidade por câncer de mama feminino em Goiânia (56,8\%), seguida pela cor parda (25,4\%). Em um estudo em Vitória, a taxa de mortalidade foi maior em muIheres de cor parda (60\%), seguida da cor branca (38\%) (ALBRECH CAM, et AL, 2013)

No presente estudo demonstrou-se que a mastectomia total foi o tipo cirúrgico mais realizado $(55,6 \%)$, seguido da cirurgia conservadora em $43,8 \%$ dos casos (Gráfico 4); o que está de acordo com estudo feito no Rio de Janeiro, o qual cita, que apesar de existir uma tendência à cirurgias conservadoras, $61,3 \%$ das mulheres também foram submetidas a mastectomia radical (HADDAD NC, et al., 2015).

Correlacionando o procedimento cirúrgico com a idade das pacientes, observou-se alta prevalência de mastectomias totais em mulheres na faixa etária de até 50 anos e redução dessa frequência com o avançar da idade (Gráfico 10). Em artigo de revisão publicado na Revista Médica Minas Gerais, foi abordado que a escolha do procedimento cirúrgico deve ser baseada na relação entre o estadio do tumor e o tamanho da mama, desconsiderando-se a idade (ALVARENGA FV, et al, 2017).

Em termos de preservação da autoestima feminina e diminuir as consequências da função sexual, a cirurgia conservadora seria a de escolha, já que também associa-se com a menor proporção de sintomas depressivos dessas mulheres, conforme estudo realizado por Moura NAV, et al. (2013) na cidade de Teresina (HADDAD NC, et al., 2015; MOURA NAV, et al., 2013; SOUZA NHA, et al., 2017).

O tipo histológico predominante no estudo foi o carcinoma tipo ductal, representando 90,5\% do total (Gráfico 5); o que é compatível com a literatura mundial.

De acordo com estudo realizado em Boston em 2015 no Instituto do Câncer, foi constatado a predominância do tipo ductal em comparação com os outros tipos, em especial o ductal in situ, que se relaciona ao aumento da prevalência com a detecção precoce da neoplasia (SAGARA Y, et al., 2015).

\section{CONCLUSÃO}

Os achados do presente estudo na qual avalia mulheres portadoras de câncer de mama submetidas a cirurgia oncológica, comprovam que o aumento de pacientes diagnosticadas com tal neoplasia é diretamente proporcional ao acréscimo da idade, sendo a idade média de 59,9 anos. Somando-se também a avaliação da raça, com prevalência significativa na população branca, e semelhança desses achados com a literatura vigente. Este estudo tem como análise adicional as características do tumor primário, do acometimento linfonodal e da presença de metástase classificados na sigla TNM, sendo de grande relevância para o diagnóstico e posterior tratamento. Por fim, a avaliação cirúrgica das mulheres estudadas demonstra que a mastectomia foi prevalente em pacientes abaixo dos 50 anos, em contraposição a cirurgia conservadora ou chamada também de setorectomia comumente realizada em idades superiores.

\section{REFERÊNCIAS}

1. A.C. CAMARGO CANCER CENTER, 2019. Câncer de mama. Disponível em: https://www.accamargo.org.br/tiposde-cancer/mama. Acesso em: 8 mar. 2019.

2. ALBRECH CAM, et al. Mortalidade por câncer de mama em hospital de referência em oncologia. Rev Brasileira epidemiologia, 2013; 16(3): 582-591.

3. AMERICAN CANCER SOCIETY. 2017. In: About Breast Cancer. Disponível em: https://www.cancer.org/content/dam/CRC/PDF/Public/8577.00.pdf. Acesso em: 15 abr. 2019.

4. American Cancer Society. 2016. In: Breast Cancer: Facts \& Figures. Disponível em: https://www.cancer.org/content/dam/cancer-org/research/cancer-facts-and-statistics/breast-cancer-facts-and-figures/breast-cancer-facts-andfigures-2015-2016.pdf. Acesso em: 20 abr. 2019.

5. AZEVEDO BA, et al. Perfil das mulheres com Câncer de mama. Rev de Enfermagem UFPE online, 2017; 11(6): 2264-72.

6. BEREK JS, NOVAK. Tratado de Ginecologia. 15.ed. Rio de Janeiro: Guanabara Koogan, 2014. 1135- 1146p.

7. BREGAGNOL RK, DIAS AS. Alterações funcionais em mulheres submetidas à cirurgia de mama com linfadenectomia axilar total. Rev Brasileira de Cancerologia, 2010; 56(1): 25-33. 
8. FERLAY J, et al. Estimate Incidence, Mortality and Prevalence Worldwide in 2012. Globocan 2012 (IARC), 2012 ; 1(11).

9. FRAZÃO A; SKABA MMFV. Mulheres com câncer de mama: as expressões da questão social durante o tratamento de quimioterapia neoadjuvante. Rev Brasileira de Cancerologia, 2013; 59(3): 427-435.

10. FREITAS F, et al. Rotinas em Ginecologia. 6aㅡ ed. Porto Alegre: Artmed, 2011; 556p.

11. HADDAD NC, et al. Perfil sociodemográfico e de saúde de mulheres submetidas à cirurgia para câncer de mama. Rev Hupe UERJ, 2015; 14: 28-35.

12. INSTITUTO NACIONAL DE CÂNCER JOSÉ ALENCAR GOMES DA SILVA, 2019.

13. MIGOWSKI A, et al. Diretrizes para detecção precoce do câncer de mama no Brasil- Novas recomendações nacionais, principais evidências e controvérsias. Caderno de Saúde Pública, 2018; 34(6): 1-16.

14. MINISTÉRIO DA SAÚDE (BR). In: Portaria no 1.008 de 30 de setembro de 2015. Disponível em: http://bvsms.saude.gov.br/bvs/saudelegis/sas/2015/prt1008_30_09_2015.html. Acesso em: 15 abr. 2019.

15. MOURA NAV et al. Perfil epidemiológico de mulheres com câncer de mama tratadas em hospital filantrópico de referência. Rev. Enfermagem UFPI, 2013; 2(4):35-41.

16. MUNHOZ CAS. Câncer de mama e organização de serviços: do atendimento básico à referência na Casa de Saúde Santa Marcelina Itaquera no município de São Paulo. Dissertação (Mestrado) - Faculdade de Saúde Pública. Universidade de São Paulo, São Paulo, 2009; 35-36p.

17. PAIVA CE, et al. Fatores de Risco para Câncer de Mama em Juiz de Fora: um estudo controle. Rev Brasileira de Cancerologia, 2002; 48(2): 231-237.

18. PAIVA CJK, CESSE EAP. Aspectos relacionados ao atraso do diagnóstico e tratamento do câncer de mama em uma unidade hospitalar de Pernambuco. Rev Brasileira de Cancerologia, 2015; 61(1): 23-30.

19. PAULINELLI RR, et al. A situação do câncer de mama em Goiás, no Brasil e no mundo: tendências atuais para a incidência e a mortalidade. Revista Brasileira de Saúde Materno Infantil, 2003; 3 (1): 17-24.

20. PINHARD CR, MERCÊS NNA. Fatores de Risco para Câncer de Mama: Nível de conhecimento dos acadêmicos de uma universidade. Rev. Enfermagem. UERJ, 2009; 17(1): 102-106.

21. PINHEIRO AB, et al. Câncer de mama em mulheres jovens: análise de 12689 casos. Revista Brasileira de Cancerologia, 2013; 59(3): 351-359.

22. SAGARA Y, et al. Survival Benefit of Breast Surgery for Low-Grade Ductal Carcinoma in Situ. JAMA Surgery, 2015; 150(8): 739-745.

23. SALIMENA AMO, et al. Mulheres enfrentando o câncer de mama. Rev Min Enferm, 2012; 16(3): 339-347.

24. SILVA FX, et al. Mamografia em mulheres assintomáticas de 40 a 49 anos. Rev. Saúde Pública, 2014; 48(6): 931939.

25. SOARES BMS, et al. Características das mulheres com câncer de mama assistidas em serviços de referência do norte de Minas Gerais. Rev Brasileira de Epidemiologia, 2012; 15(3): 595-604.

26. SOUZA NHA, et al. Câncer de mama em mulheres jovens: estudo epidemiológico no nordeste brasileiro. Sanare, $2017 ; 16(2): 60-67$.

27. TESSARO S. et al. Contraceptivos orais e câncer de mama: estudo de casos e controles. Revista de Saúde Pública, $2001 ; 35(1): 32-38$.

28. THULER LCS, MENDONÇA GA. Estadiamento inicial dos casos de câncer de mama e colo do útero em mulheres brasileiras. Rev Bras Ginecol Obstet, 2005; 27(11): 656-60.

29. Urban LABD, et al. Breast cancer screening: updated recommendations of the brazilian college of radiology and diagnostic imaging, brazilian breast disease society, and brazilian federation of gynecological and obstetrical associations. Mastology, 2017; 27(3): 258-64. 